\title{
Extensive functional evaluations to monitor aerobic training in Becker Muscular Dystrophy: A case report
}

\author{
Caterina Tramonti, Bruno Rossi, Carmelo Chisari \\ Unit of Neurorehabilitation, Department of Neuroscience, University Hospital of Pisa, Italy \\ This article is distributed under the terms of the Creative Commons Attribution Noncommercial License (CC BY-NC 4.0) which \\ permits any noncommercial use, distribution, and reproduction in any medium, provided the original author(s) and source are credited.
}

\begin{abstract}
Low-intensity aerobic training seems to have positive effects on muscle strength, endurance and fatigue in Becker Muscular Dystrophy (BMD) patients. We describe the case of a 33-year old BMD man, who performed a four-week aerobic training. Extensive functional evaluations were executed to monitor the efficacy of the rehabilitative treatment. Results evidenced an increased force exertion and an improvement in muscle contraction during sustained exercise. An improvement of walk velocity, together with agility, endurance capacity and oxygen consumption during exercise was observed. Moreover, an enhanced metabolic efficiency was evidenced, as shown by reduced lactate blood levels after training. Interestingly, CK showed higher levels after the training protocol, revealing possible muscle damage. In conclusion, aerobic training may represent an effective method improving exercise performance, functional status and metabolic efficiency. Anyway, a careful functional assessment should be taken into account as a useful approach in the management of the disease's rehabilitative treatment.
\end{abstract}

Key Words: Becker Muscular Dystrophy, Oxidative Metabolism, Aerobic Training, Muscle Damage

Eur J Transl Myol 2016; 26 (2): 81-86

Becker Muscular Dystrophy (BMD) is an X-linked inherited muscle disease affecting the proximal muscles of the upper and lower limbs. The pathogenic mechanism is represented by mutations in gene encoding dystrophin, a cytoplasmic protein involved in the dystrophin glycoprotein complex (DGC), connecting the extracellular matrix to the intracellular cytoskeleton at the sarcolemma. Therefore, the loss of dystrophin results in an instability and disruption of the sarcolemma. In general, BMD patients have an in-frame mutation resulting in aberrant but partially functional protein, thus representing a milder variance of the Duchenne Muscular Dystrophy (DMD). ${ }^{1} \quad$ Taking these considerations into account, physical activity in BMD has always been avoided, because of the potential harmful effects due to mechanical stress on the sarcolemma during muscle contraction. ${ }^{2}$ Generally, patients with dystrophies have been advised not to perform high intensity training and eccentric muscular activity. In fact, different trials suggested to prefer lowresistance training, even for the positive effects on metabolic enzyme activities and possibly modulatory influences on the composition of isoforms of contractile proteins. ${ }^{3}$ Furthermore, a recent trial evidenced that lowintensity aerobic exercise seems to have positive effects on muscle strength, endurance and fatigue, with no deleterious effects on BMD patients. ${ }^{4}$

We monitored the benefits and safety of a specific aerobic training in a 33-year-old man diagnosed with BMD, suffering from excessive daily fatigability and proximal lower limb muscle impairment. Our main purpose was to reveal both the potential benefits and the possible harmful effects of the aerobic training by means of extensive functional evaluations.

\section{Case Report}

We describe the case of a 33-year-old BMD patient, who underwent a 4-week aerobic training performed on a treadmill. He reports the onset of frequent falls when he was four years-old. At five years-old, he performed a muscle biopsy and was diagnosed with Becker Muscular Dystrophy. He carries out regular neurological, cardiological and pneumological controls. He is substantially independent in activities of daily living. In the last year, he refers a worsening of the clinical condition, with increased fatigability and difficulty in climbing stairs. A recent muscle Magnetic Resonance (RM) of the lower limb evidenced: "presence of fatty replacement of muscle bellies in the gluteus and tensor fascia lata, bilaterally. Moreover, a 


\section{Monitor aerobic training in Becker Muscular Dystrophy}

Eur J Transl Myol 26 (2): 81-86

marked fatty infiltration interested the thigh muscles bilaterally", Fig.1. His anthropometric data are: height $1,70 \mathrm{~m}$, weight $78 \mathrm{Kg}$, BMI 27. The subject gave his written informed consent before the training protocol.

\section{Methods}

Assessment

\section{Muscle strength}

The lower limbs strength was evaluated by means of manual muscle testing using the Medical Research Council scale (MRC): ${ }^{5}$ he was scored 3 at hip and knee extension and 4 at knee flexion and ankle dorsiflexion, bilaterally.

Moreover, knee and hip extension together with ankle dorsiflexion muscle strength was assessed measuring the maximum voluntary contraction (MVC), in isometric condition, on an isokinetic dynamometer (PrimusRS Multi-Joint System dynamometer, BTE Technologies).

For the evaluation of knee extension the patient sat on a chair with his upper body strapped firmly against the backrest of the chair, straps tightened across his thighs and arms folded. Hip and knee angles were set respectively at $110^{\circ}$ and at $120^{\circ}\left(180^{\circ}\right.$ refers to full extension). ${ }^{6}$ Hip extension was assessed in standing position, with the instantaneous axis of rotation straight across from the greater trochanter to the axis of the dynamometer. The thigh stabiliser strip routinely used was positioned just proximal to the knee joint. The hip angle was set at $160^{\circ}$. Ankle dorsiflexion was evaluated with the subject lying in a supine position, with hip and knee in full extension. Patient's position was secured by pelvic, thigh and leg stabilizer belts. Location of footplate was adjusted according to the patient's height. Patient's foot was strapped on the footplate, and the ankle angle was set at $150^{\circ} .^{7}$ Before starting the test sections, the subject was instructed about the correct procedure to exert maximum voluntary contraction, as hard as possible without moving the rest of the body, and to maintain it for $3 \mathrm{~s}$. Three consecutive repetitions for both sides were performed. A rest time (10 s) was observed between consecutive trials. The three tests were accepted when the coefficient of variation $(\mathrm{CV})$ was less than $10.0 \% .^{6}$

Muscle fatigue

Muscle fatigue was evaluated measuring sustained contractions in isometric tasks in lower limbs (hip extension and ankle dorsiflexion) on an isokinetic dynamometer. Fatigue during sustained task in knee extension was not evaluated, because of the known limitations of the method to assess very weak muscles. The subject was instructed to exert maximum voluntary contraction for hip extension and for ankle dorsiflexion, and then to maintain it for $60 \mathrm{~s}$ without any further encourage during the performance. Both sides were evaluated. An index of muscle fatigue was extracted as the slope of linear regression of contraction trend ${ }^{8}$ For each trial it was evaluated the percentage of the decrease, describing the variation between the first and the last second of the force exerted, using the following formula: Decr [\%]: (yfinal-yinitial/yinitial).

\section{Functional Scales}

Gait velocity, agility and endurance were assessed by means of Ten Meters Walking Test (10MWT), Timed Up and Go Test (TUG) and Six Minutes Walking Test (6MWT), respectively. ${ }^{9-11}$ An estimate of the oxygen consumption following the submaximal exercise was provided by the Physiological Cost Index (PCI), which corresponds to the increase in heart rate per meter walked by the subject. ${ }^{12}$

Muscle oxidative efficiency

Muscle oxidative efficiency was indirectly evaluated by means of assay of blood lactate levels before and after an incremental sub-maximal aerobic exercise on a treadmill. Lactate was assessed, through venous blood samples, collected before (T0), immediately after (T1) and 5, 10 and 30 minutes after the end of the exercise (respectively: T2, T3, T4). ${ }^{6}$ The subject was asked to rest for thirty minutes before collecting basal blood samples.

\section{The susceptibility to myofibral damage}

The susceptibility to myofibral damage was evaluated through the dosage of creatine-phospho-kinase (CK) serum level before and after a walking test, collecting venous blood samples at rest and at 1, 3, 6 and $24 \mathrm{~h}$ after the end of the exercise. ${ }^{6}$

\section{Training protocol}

The patient underwent an aerobic training protocol, consisting of walking on a treadmill at a maximal tolerable speed. The rehabilitative treatment lasted 4 weeks, with a frequency of 3 days/week. Prior to and after it all the aforementioned evaluations were performed.

Heart rate was constantly monitored during

the exercise. The speed was gradually increased in the first minute of exercise, reaching the maximum one tolerated by the patient. It was then maintained/modified during walk to let the patient maintaining heart rate (HR) between $65-80 \%$ of the theoretically calculated maximal value, thus keeping the exercise in predominantly aerobic conditions. When the HR exceeded the $80 \%$, the speed was

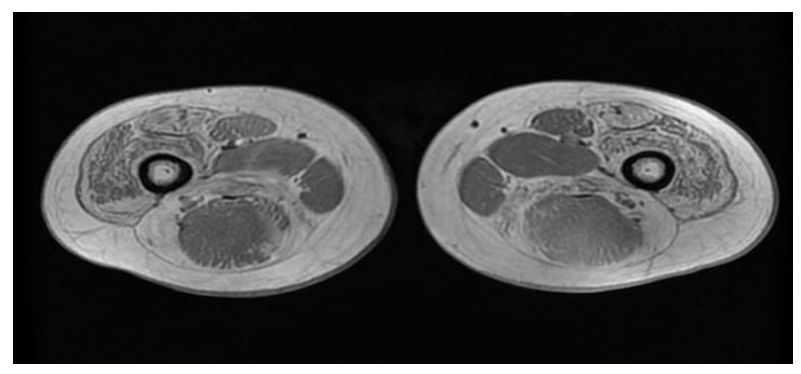

Fig.1 T1 weighted MRI of thigh muscles performed before the training program. 


\section{2.a}

\section{Knee Extension}

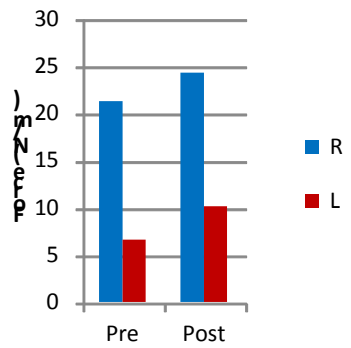

2.d

Fatigue right hip

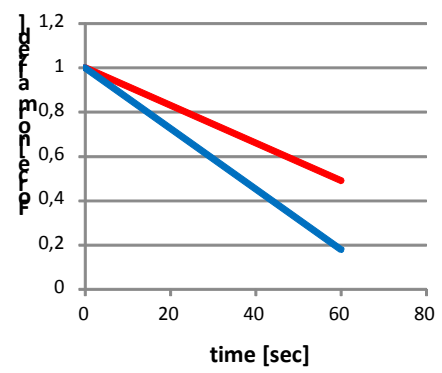

2.b

2.c

\section{Hip Extension}

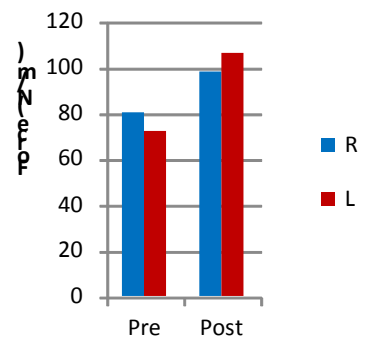

\section{Ankle Dorsiflexion}

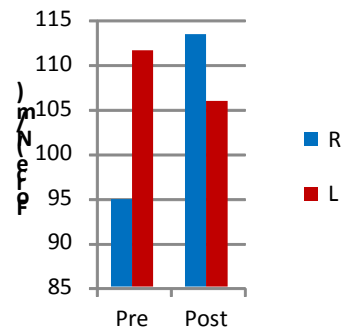

2.e

Fatigue right ankle

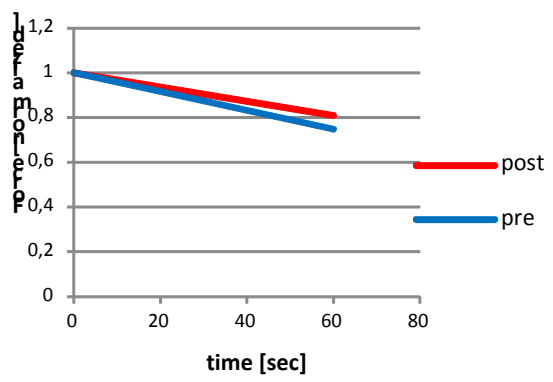

Fig. 2 Maximal voluntary contraction at knee extension (2.a), hip extension (2.b) and ankle dorsiflexion (2.c) before and after training. Fatigue during isometric task at right hip extension (2.d) and at right ankle dorsiflexion (2.e) before and after training

gradually slow down, until HR returned in the aerobic range. The slope of the treadmill was not increased. The patient was encouraged to walk at least $20 \mathrm{~min}$ or more in each session. ${ }^{6}$ All sessions were performed into an intra-hospital gymnasium and monitored by a physical therapist.

\section{Results}

Considering force evaluation during isometric contractions, we observed an exerted strength lower than the normal range in both limbs. After the rehabilitative protocol, an increase in strength was observed in knee ( 21,3 vs $24,3 \mathrm{~N},+14,08 \%$ and 6,6 vs $10,12 \mathrm{~N},+53,33 \%$, right and left respectively, Fig. 2.a), hip extensor muscles $(80,1$ vs $98 \mathrm{~N},+22,34 \%$ and 72,1 vs $106,1 \mathrm{~N}$, $+47,15 \%$ right and left respectively, Fig. 2.b) and ankle dorsiflexor at the right side $(94,8$ vs $113,3 \mathrm{~N},+19,51 \%$, Fig. 2.c). Before training, at the end of the fatiguing test a decrease in force exertion in hip extension (-67,33 and $-22,66 \%$ right and left respectively) and in ankle dorsiflexion $(-26,28$ and $-43,50 \%$, right and left respectively) was observed. After the rehabilitative training, results evidenced a reduction in the decrement of muscle force exerted in hip extension at the right side $(-57,11 \%$, Fig. 2.d), and in ankle dorsiflexion bilaterally $(-15,84 \%$, right side, and $-28,73 \%$, left side, Fig. 2.e). Functional tests showed an improvement of walk velocity (10MWT improved from 1,4 m/s to 1,6 $\mathrm{m} / \mathrm{s}$, Fig. 3.a), together with walking agility and endurance, as shown by the results of TUG test and 6MWT (Fig. 3.b, Fig. 3.c). Before training the analysis of the physiological cost index revealed a value of 0,28 , which was improved after the aerobic protocol (28,57\%), as evidenced in Fig. 3.d.

Before the treatment, lactate assay showed resting values higher than normal range $(4,28 \mathrm{mmol} / \mathrm{L}$, n.v.: $0,5-2,19 \mathrm{mmol} / \mathrm{L})$. Lactate levels revealed a decrease at the end of the exercise and at 10 and 30 min during the recovery period. After the rehabilitative treatment, lactate showed a decreased resting level compared to the values assessed before training; moreover, lower levels at the end of the exercise and at 5,10 and $30 \mathrm{~min}$ during the recovery period were observed (Fig. 3.e). Before training, CK assay showed resting values higher than normal range (532 U/L, n.v. < 190 U/L). After the exercise test, CK levels increased at 1, 3, 6, 


\section{Monitor aerobic training in Becker Muscular Dystrophy}

Eur J Transl Myol 26 (2): 81-86

3.b

10MWT

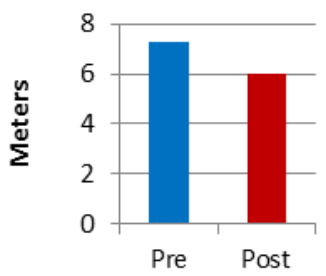

3.e 3.c

3.d
TUG

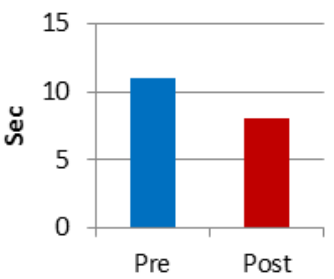

6MWT

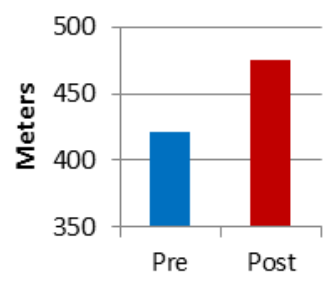

$\mathrm{PCl}$

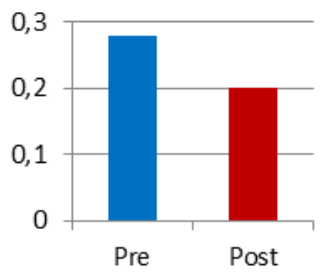

3.f

Lactate curve

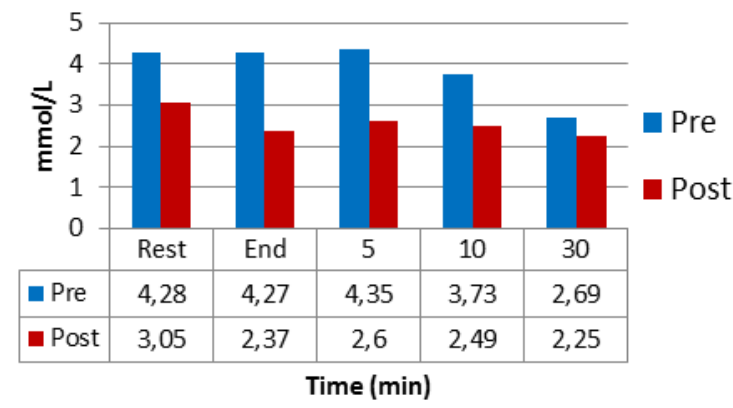

CPK curve

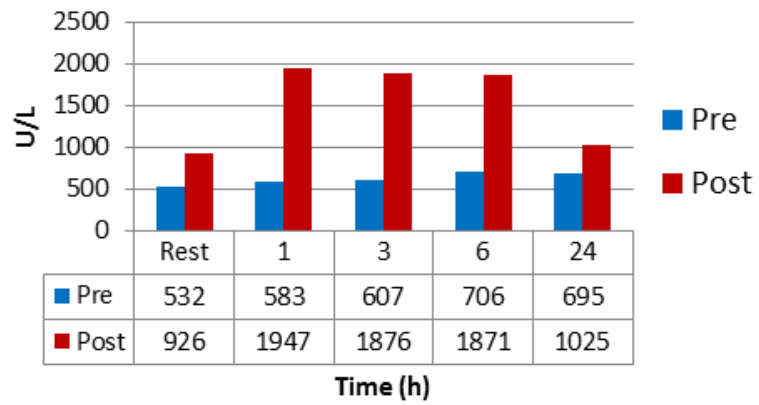

Fig 3. Ten Meter Walking Test (10MWT, 3.a), Timed Up and Go test (TUG, 3.b), Six Minute Walk Test (6MWT, 3.c) and Physiological cost index (PCI, 3.d) before and after training. Lactate curve (3.e) and creatine-phosphokinase curve $(C K, 3 . f)$ before and after training.

24 hours during recovery period, respectively. After the aerobic training, CK levels at baseline were increased compared to values assessed before training (926 U/L). Furthermore, CK levels showed a further increase at 1, 3, 6, 24 hours after the exercise test (Fig. 3.f).

\section{Discussion}

Patients with dystrophinopathies have always been advised not to perform high-resistance and eccentric exercise protocols, ${ }^{2}$ even if different studies indicate that muscle training may be beneficial for these patients, especially at the early phases of the disease. ${ }^{3}$

In this context, we tested the effects of a 4-week aerobic training in a young BMD patient, performing different functional evaluations, in order to test the widespread benefits on force and fatigue exertion, walking abilities, endurance capacity, metabolic efficiency and exerciseinduced myofiber damage.

Our results showed an increase in strength in knee and hip extension, while the evaluation of muscle fatigue during sustained contraction evidenced an improvement both at hip extension in the right side and at ankle dorsiflexion bilaterally. These data are of quite importance, as far as it was demonstrated that a reduction in force exertion could correlate with poorer functional abilities in daily living. ${ }^{13}$ Secondly, an improvement in the Timed Up and Go and in the 6Minutes Walking Test was observed, suggesting amelioration in dexterity and endurance capacities. The relevancy of these data lies in the evidence that patients' poor performance in these tests is related to greater balance problems, ${ }^{14}$ and to changes in walking function. ${ }^{15}$ Therefore, what is remarkable is the fact that after four weeks of aerobic training the patient improved his endurance and ability to perform postural transfers, thus increasing his functional capacities. Moreover, the improvement in the 6MWT was associated with a decrease of Physiological Cost Index (PCI), a clinically accessible measure of oxygen consumption during walking. ${ }^{16}$ As far as an increased physical strain is a common problem in subjects with motor disorders, ${ }^{17}$ an improvement of the energy cost during walking might provide an additional marker of better ambulatory function in our patient.

The analysis of metabolic efficiency evidenced abnormal lactate levels at baseline. Lactate curve did not evidence an increase in serum levels at the end of the exercise and at any time during recovery period, both before and after the training, although revealing values above the normal range. The impairment of the muscle oxidative pathway determines a precocious switch to the lactic anaerobic metabolism during a 


\section{Monitor aerobic training in Becker Muscular Dystrophy}

Eur J Transl Myol 26 (2): 81-86

prolonged exercise, so that the lactacidemia can represent an indirect index of muscle oxidative capacity. Recent studies in animal models of DMD suggest that muscular dystrophy alters muscle energetics and mithocondrial dynamics. Moreover, multiple metabolic adaptations are induced to maintain energy homeostasis in dystrophic muscle. Interestingly, it was also revealed more oxygen consumption relative to force produced, suggesting an higher metabolic demand. A potential explanation for this higher oxygen consumption could be due to either an inefficiency in energy utilization or a decrease in energy production by mitochondria. In the end, the extreme metabolic stress may lead the diseased muscle to rely on increased glycolysis to meet energy demands. ${ }^{18}$ Therefore, we can postulate an impaired oxidative metabolism efficiency in our patient, even during normal activities of daily living. Nevertheless, the reduction in lactate levels after the submaximal test seems quite controversial, even if recent studies evidenced that during moderate- intense exercise lactate might be oxidized by oxidative muscle fibers. ${ }^{19}$ Anyway, the slight decrease in lactate levels at baseline and after the exercise test could evidence an amelioration in his muscle aerobic capacity after the aerobic training.

These findings are quite in line with a recent trial performed on eleven BMD patients by Sveen et al. (2008). ${ }^{4}$ The benefits of a 12 -week moderate-intensity aerobic training was revealed by increased work capacity with self-reported improvements in endurance, leg muscle strength and walking distance. Moreover, plasma lactate levels and heart rate at rest and at exhaustion tended to be lower when assessed after training, with no muscle damage inflicted as reflected by unchanged CK levels during training. On the same line, Roque et al. $(2010)^{19}$ evaluated the effect of a 5-month lasting aerobic program on a treadmill in a young adult BMD patient, underling an increase in quadriceps muscle strength, along with an increased aerobic capacity after the training period. ${ }^{20}$

However, besides the aforementioned benefits and contrary to what emerged in previous trials, interestingly we found a rise of CK serum levels at rest and after the submaximal test. As known, plasma CK analysis is commonly used as safety marker of muscle damage in muscular dystrophies. ${ }^{21}$ Therefore, we cannot disregard that besides an improvement in functional status of this patient, the susceptibility to exercise-induced muscle damage after the rehabilitative protocol worsened. Therefore, it seemed interestingly to underline that besides functional improvement induced by training, we must be careful to monitor the rehabilitative intervention in order to avoid possible side effects. Potential limitations are consistent with the fact that we described only one patient, therefore we cannot argue extensive conclusions. Moreover, the patient presented baseline CK levels higher than normal values, therefore assuming that his muscle impairment was already evident in the normal activity of daily living. In conclusion, we propose to continue and extend this approach to a larger sample size, in order to provide more solid data. This study support the actual approach to the management of patients with BMD, which should involve frequent periods of low to moderate aerobic exercise. Anyway, assessing functional status with specific evaluations should be considered as an important tool to direct and monitor the safety of rehabilitative interventions.

\section{Acknowledgement}

We would like to thank Physiotherapist, Dr. Chiara Simonella, who assisted the patient during the treatment.

\section{Authors' contributions}

CT performed functional evaluations, contributed to data analysis and interpreted the results. BR supervised the manuscript. CC designed the study protocol and contributed to interpretation of data and to manuscript supervision. All authors read and approved the final version of the manuscript.

\section{Conflict of Interest}

The authors declare no conflict of interests, since they did not received any funding for the present research.

\section{Corresponding Author}

Carmelo Chisari, Unit of Neurorehabilitation, Department of Neuroscience, University Hospital of Pisa Via Paradisa 2, 56126, Pisa, Italy. Tel.: +39 (0) 50 996907, Fax: +39 (0) 50995430.

E-mail: c.chisari@ao-pisa.toscana.it

E-mail of coauthors

Caterina Tramonti: caterina.tramonti@gmail.com Bruno Rossi: b.rossi@ao-pisa.toscana.it

\section{References}

1. Allikian MJ, McNally EM. Processing and Assembly of the Dystrophin Glycoprotein Complex. Traffic 2007;8:177-83.

2. Petrof BJ. The molecular basis of activityinduced muscle injury in Duchenne muscular dystrophy. Mol Cell Biochem 1998;179:111-23.

3. Ansved T. Muscle training in muscular dystrophies. Acta Physiol Scand 2001;171:35966.

4. Sveen ML, Jeppesen TD, Hauerslev S, Kober L, Krag TO. Vissing J. Endurance training improves fitness and strength in patients with Becker muscular dystrophy. Brain 2008;131:2824-3

5. Medical Research Council of the United Kingdom. Aiak to Examination of the Peripheral Nervous System: Memorandum No 45. Palo Alto, Calif: Pedragon House; 1978.

6. Dalise S, Bertolucci F, Simonella C, Rossi B, Chisari C. Intensive aerobic training improves motor performances and oxidative metabolism 


\section{Monitor aerobic training in Becker Muscular Dystrophy}

Eur J Transl Myol 26 (2): 81-86

efficiency in chronic polymyositis: A case report. Neuromuscul Disord 2012;22:221-5. doi: 10.1016/j.nmd.2012.10.015.

7. Özyemİşcİ Taşkıran O, Özdoğan V, Sepİcİ V, Meray J. Test-Retest and Inter-Rater Reliability of Isokinetic ankle Dorsiflexor and Plantar Flexor Strength Measurement in Healthy Adults. Turk J Phys Med Rehab 2013;59:32-5.

8. Zupan A, Gregorič M , Valenčič V , Vandot S.Effects of Electrical Stimulation on Muscles of Children with Duchenne and Becker Muscular Dystrophy. Neuropediatrics 1993;24:189-92.

9. Morganti B, Scivoletto G, Ditunno P, Ditunno J, Molinari M. Walking index for spinal cord injury (WISCI): criterion validation. Spinal Cord 2005;43:27-33.

10. Schoene D, Wu SM, Mikolaizak AS, Menant JC, Smith ST, Delbaere K, Lord SR. Discriminative ability and predictive validity of the timed up and go test in identifying older people who fall: systematic review and meta-analysis. J Am Geriatr Soc 2013;61(2):202-8.

11. Kempen JC, Harlaar J, Van der Kooi AJ, De Groot IJ, Van den Bergen JC, Niks EH, Verschuuren JJ, Brehm MA. Reliability of the walking energy cost test and the six-minute walk test in boys with Duchenne muscular dystrophy. Neuromuscul Disord 2014;24:216-21.

12. MacGragor J. The evaluation of patient performance using long-term ambulatory monitoring technique in the domiciliary environment. Physiotherapy 1981;67:30-3.

13. Uchikawa K, Liu M, Hanayama K, Tsuji T, Fujiwara T, Chino N. Functional status and muscle strength in people with Duchenne muscular dystrophy living in the community. J Rehabil Med 2004;36:124-9.
14. Aras B, Aras O, Karaduman A. Reliability of balance tests in children with Duchenne muscular dystrophy. Scientific Research and Essays 2011;20:4428-31.

15. McDonald CM, Henricson EK, Han JJ, Abresch RT, Nicorici A, Atkinson L, Elfring GL, Reha A, Miller LL. The 6-minute walk test in Duchenne/Becker muscular dystrophy: longitudinal observations. Muscle Nerve 2010;42:966-74.

16. Fredrickson E, Ruff RL, Janis J, Daly JJ. Physiological Cost Index as a Proxy Measure for the Oxygen Cost of Gait in Stroke Patients. Neural Repair 2007;2:429-34.

17. Waters RL, Mulroy S. The energy expenditure of normal and pathologic gait. Gait Posture 1999;9:207-31.

18. Pant M, Sopariwala DH, Bal NC, et al. Metabolic Dysfunction and Altered Mitochondrial Dynamics in the Utrophin-Dystrophin Deficient Mouse Model of Duchenne Muscular Dystrophy. PLOSone 2015;10:1-19.

19. Gladden LB. Lactate metabolism: a new paradigm for the third millennium. J. Appl. Physiol 2004;558:5-30.

20. Roque JM, Carvalho VO, Pascoalino LN, Ferreira SA, Bocchi EA, Guimarães GV. Physical Training in Becker Muscular Dystrophy Associated with Heart Failure. Arq Bras Cardiol 2011;97:128-31.

21. Baird MF, Graham SM, Baker JS, Bickerstaff GF. Creatine-Kinase- and Exercise-Related Muscle Damage Implications for Muscle Performance and Recovery. Journal of Nutrition and Metabolism 2012. pp 1-13. 